\title{
Ia importancia de América Latina en el sistema internacional en la década de los ochenta: un ensayo bibliográfico
}

I. INTRODUCGIÓN: EL CONTEXTO INTERNACIONAL Y REGIONAL

La creciente importancia de América Latina en el sistema internacional se manifiesta en un contexto caracterizado por el surgimiento de nuevas tendencias en el sistema internacional, por los rápidos cambios que ocurren en él, por la complejidad que éste va adquiriendo, y por el impacto que el mismo tiene cada vez más en el desarrollo de los Estados-Naciones de la región. En breve, los cambios y tendencias que se pueden observar son los siguientes:

a) Se aprecia una creciente interdependencia en el sistema internacional que involucra prácticamente a todos los Estados-Naciones. Fenómenos nacionales e internacionales (e. g., elecciones, revoluciones, conflictos interestatales o intraestatales, crisis energética, narcotráfico, cambios tecnológicos, crisis económico-financieras, deuda, venta de armas, etc.) que ocurren en cualquier Iugar del globo son conocidos inmediatamente en sitios lejanos y no tardan en afectar el comportamiento de gobiernos y de otros "actores" nacionales e internacionales. Muchos de los problemas que confrontan nuestras sociedades (coyunturales o estructurales) tienen su origen o están vinculados con eventos, crisis, problemas o estructuras internacionales. El mundo parece mucho más interconectado y ya no existen problemas importantes que no afecten a todos de alguna manera; nadie es totalmente invulnerable. Tampoco esos problemas se pueden resolver unilateralmente; y se da así una interdependencia de problemas y soluciones.

b) Esa interdependencia también se observa en la creciente proliferación de grupos, intereses o "actores" internacionales - tanto privados como estatales- que van creando una red cada vez más compleja de interacción e intercambio internacional; formando regimenes o circuitos globales con sus propias reglas y comportamientos en lo económico-financiero, en lo educativo-cultural, en lo cientifico-tecnológico, en lo político, en lo militar, etc. Estos desarrollos 
van transnacionalizando o internacionalizando las sociedades nacionales, y se ven facilitados por el aumento, diversificación, y rapidez de las comunicaciones.

c) Se exhibe una creciente multipolaridad en el sistema internacional, con el surgimiento de nuevos centros de poder económico, tecnológico, o financiero; acompañado de una disminución de la hegemonf́a monolítica de las dos superpotencias y de una nueva distensión entre ellas (¿una nueva detente?). que va creando mayores espaciós políticos y económicos para paises intermedios.

d) En los países del "Norte" se percibe una creciente atención a la demanda de los países en desarrollo por un nuevo orden económico internacional más equitativo y justo, donde los costos y beneficios de la economía mundial sean mejores distribuidos. Hay por por lo menos una nueva conciencia en e] "Norte" de que su bienestar está vinculado e trechamente a estós cambios solicitados y a Ja estabilidad y bienestar del "Sur". Esto abre nuevas posibilidades y nuevos desafíos para los países en desarrollo.

Pero la relevancia adquirida recientemente por América Latina y el Caribe, se debe también a la nueva importancia y a la mayor actuación de algunos países de la región; en numerosos y significativos temas y problemas internacionales ${ }^{1}$.

Brevemente, por ejemplo, la política exterior norteamericana, al ubicar el conflicto centroamericano en el eje Este-Oeste y percibir la crisis como un caso de amenaza a su seguridad nacional, le ha conferido a Ia región una alta importancia estratégica en términos de las relaciones Este-Oeste. Es así como los países de Centroamérica, apoyados por el grupo de los ocho (Grupo de Contadora y Grupo. de Apoyo: Colombia, México, Panamá y Venezuela/Argentina, Brasil, Perú y Uruguay), y Guba se han convertido en los últimos años en importantes actores de un conflicto que, aunque de naturaleza fundamentalmente regional, podrfa eventualmente afectar el balance globa ${ }^{2}$. Por otro lado, la importancia geopolítica de América

'Joseph Grunwald (ed.), Latin America and the World Economy. A Changing International Order (Sage Publications, 1978); Wolf Grabendorff y Riordan Roett (eds.), Latin America, Western Europe, and the United States (Praeger, I985); J. Lincoln y E. Ferris (eds.), The Dynamics of Latin American Foreign Policies (Westview, 1984); A. Krieger Vasena y Javier Pazos, Latin America. A Broader World Role (London: E. Benn Ld., 1973); E. Hill y L. Tomassini (comp.), América Latina y el Nuevo Orden Económico Internacional (Buenos Aires: Editorial:Belgrano, 1982); L. Tomassini (ed.), El Didlogo Norte-Sur, Una Perspectiva Latinoamericana (Buerios Aires: Editorial Belgrano, 1982); $\mathrm{H}$. Jaguaribe (ed.), La Politica Internacional de los Años 80 (Buenos Aires: Editorial Belgrano, 1982)..

${ }^{2} \mathrm{La}$ importancia estratégica de una región o pafs en el sistema de relaciones internacionales aquí se entiende en términos relativos; o sea, su importancia se puede evaluar en términos de lo que esa región o país significa comparativamente en relación a los actores más poderosos del sistema internacional; en el 
Central y el Caribe, está condicionada por la proximidad geográfica de la región, la historia de las relaciones hegemónicas con ella, y por el deseo de mantener el balance de poder prevaleciente en él sistema internacional ${ }^{3}$.

Pero cabe destacar que la importancia o "peso" de esos países latinoamericanos ( $y$ aquí se debe resaltar especialmente el crucial liderazgo del Gobierno de Costa Rica) se evidencia también en el hecho de que, hasta el momento, esos países han conseguido contener a un mínimo el conflicto militar, reduciendo así las probabilidades de lo que podría llegar a ser un eventual enfrentamiento Este-Oeste (aunque sea vía terceros) de consecuencias imprevisibles para la regióñ ${ }^{4}$.

En este contexto, es imprescindible también señalar que, para Estados Unidos, América Latina representa, potencialmente, un im: portante aliado de Occidente en el conflicto ideológico con los países del bloque socialista. Pero esta importancia sólo adquirirá su real valor y peso cuando América Latina comparta efectivamente la prosperidad de Occidente y sus democracias se hayan consolidado. Recién entonces la región tendrá la misma preocupación que Estados Unidos por la seguridad del Hemisferio y podrá jugar un rol más significativo en la defensa de los intereses y valores de Occidente en el sistema internacional.

Por otro lado, América Latina, a través de los paíseș de mayor desarrollo del área, ha tenido un papel crucial en casi todos los temas o cuestiones de negociación Norte-Sur. Tanto de protagonista

caso de América Latina es obviamente en relación a la potencia hemisférica. Esto no implica, sin embargo que cada país o región no tenga en st mișma una importancia intrínseca y absoluta por sus valores históricos y culturales. En relación a la importancia estratégica de América Latina, vale la pena mencionar, sin embargo, que los pais'es latinoamericanos han resistido la noción de ubicar el conflicto centroamericano en el contexto de las relaciones Este-Oeste.

"Además de los trabajos que se tratan aquí sobre iel tema de las percepciones, ver tambien James Kurth, "The United States, Latin America and the World: The Changing International Context of us-Latin American Relations". Washington, D. C., The WiIson Center for International Scholars, Latin American Program, No 148, 1984.

«El Plan de Paz 'firmado' en Guatemala el 7 de agosto de 1987, por los cinco países centroamericanos, representa en cierta manera haber logrado resistir la presión norteamericana para imponer su perspectiva y fórmula de solución en la región. Significa ádemás haber encontrado una solución latinoamericana al problema centroamericano, ignorarido tácitamente el plan de paz propuesto poi la Administración Reagan dos dís antes de la reunión de Presidentes en Guatemala. Esta "marginalización" de Estados Unidos, es un indicio más de lo que algunos latinoamericanistas norteamericanos han venido señalando como la creciente incapacidad de los gobiernos norteamericanos de imponer automáticamerste soluciones basadas en presunciones hegemónicas anticuadas, enmaitcadas en el conflicto militar-estratégico Este-Oeste. Ver J. Kurth, op. cit, y J. Lincoln y E. Ferris (eds), The Dynamies of Latin American Foreign Policies, op. cit. 
central en el tema de la deuda externa, como de propulsor de ideas de cambio para un nuevo orden económico internacional, América Latina hoy exhibe una creciente importancia en el sistema internacional. Su capacidad de negociación en torno a la deuda, paradójicamente, se ha visto aumentada a medida que crece su endeudamiento (Us\$ 450 mil millones); $y$ sus demandas para una solución justa al problema de la deuda, (expresadas en foro de negociacions internacionales como el Fondo Monetario Internacional, el Banco Mundial, el Banco Interamericano de Desarrollo; el Diálogo NorteSur, las conferencias sobre Comercio y Desarrollo de las Naciones Unidas, la GEPAL, la OEA), así como là amenáza latente de una moratoria, tienen profundas implicaciones de cambios en temas tam cruciales e interdependientes como el de proteccionismo, el de movimiento de capitales, el de términos de intercambio, el de transferencia de tecnologias, el de tasas de interés, el del rol de multinacionales $y$ de organismos financieros internacionales; todos ellos factores vitales para la estabilidad y prosperidad del sistema económico capitalista de Occidente 5 . Pero lo que aquí resulta importante subrayar es que estas demandas latinoamericanas representan, en gran medida, las preocupaciones e intereses de la mayoría de los paises en desarrollo en las negociaciones Norte-Sur; particularmente en el contexto de la búsqueda de un nuevo orden económico internacional más equitativo.

La importancia y "peso" de América Latina en el sistema internacional se evidencia además en el hecho de que algunos de los países de mayor desarrollo de América Latina han demostrado un creciente activismo en torno a numerosos y significativos asuntos internacionales. Su presencia y liderazgo han quedado marcados en foros de negociaciones sobre temas tan trascendente; para el sistema internacional como el derecho del mar, la política contra la proliferación de armas nucleares, la del no-alineamiento, la protección de la Antártica, la !ucha contra el narcotráfico y otros.

Tampoco se debe ignorar el hecho de que América Latina, como región, ha experim`ntado en. los último; 25-30 años importantes avances en su inserción internacional, a pe:ar de la presente crisis

${ }^{5} \mathrm{La}$ participación de la deuda latinoamericana en la deuda total mundial aumentó de $39.9 \%$ en 1970 a $43.3 \%$ en 1982 . La amenaza latente de una moratoria tiene serias implicaciones para la estabilidad económica financiera del sistema capitalista occidental, como lo revela el hecho de la enorme-exposición de los principales bancos norteamericanos. Sólo la deuda de los tres paises más endeudados de América Latina representa entre el 143 y $180 \%$ de los valores de los siete bancos más importantes de Estados Unidos. Ver Carlos Massad, "External Financing in Latin America: Development Problems and Options", en: K. Middlebrook y Carlos Rico (eds.), The United States and Latin America in the 1950's. Contending perspectives on a decade of crisis (Pittsburgh, Pa.; University of Pittsburgh Press, 1986). 
de los años 80 , que incluye deuda, recesión, pérdida de mercados, empeoramiento de los términos de intercambio, etc. Por ejemplo, su Producto Bruto Interno (PBI) pasó de us\$2Il mil millones en 1960 a us $\$ 600 \mathrm{mil}$ millones en 1980; o sea, un crecimiento de $200 \%$. En términos relativos, esto significó un aumento de la participación latinoamericana en la economía mundial del $4,2 \%$ en 1960 a $5,2 \%$ en 1982. Igualmente, el ingreso per capita de América Latina exhibió un importante crecimiento, pasando de us\$1.053 en 1960 a us\$ 1.900 en 1980; lo que representó un aumento de $80 \%$, que sin embargo fue revertido en 1984 debido a la crisis, cuando el ingreso per capita cayó a us $\$ 1.750$. Con referencia a sus exportaciones al mundo, éstas crecieron de us\$9,5 mil millones en 1960 a us\$100 mil millones en 1980, lo que significó un aumento de $900 \%$. Lo mismo, prácticamente, ocurrió con sus importaciones del mundo.

Como resultado de esos cambios y nuevas realidades, a principios de la década de los 80 América Latina, por una parte, parecía amenazar con su deuda y su pos ble moratoria al sistema económico-financiero internacional; y por otra, parecía desafiar, con un aumento de la tensión política y militar en Centroamérica, la hegemonía de la potencia occidental en un área tradicionalmente considerada por ella como su indiscutible zona de influencia. En este contexto internacional aparentemente en recomposición, cabe preguntarse, por lo tanto, si en efecto América Latina está demostrando una nueva capacidad de influencia en el sistema internacional y así está adquiriendo un papel más importante en el mismo.

Teniendo lo anterior en consideración, el presente ensayo bibliográfico tiene por propósito identificar cuáles son los cambios en la importancia de América Latina percibidos por Ios destacados autores aquí incluidos, y qué relevancia le asignan a América Latina en este nuevo contexto.

\section{Los TEXTOS CONSIDERADOS}

El libro de Lars Schoultz trata dos temas poco analizados en el área del estudio de las relaciones internacionales de América Latina:

a) La importancia de América Latina en términos estratégico-mili-

\footnotetext{
'Estas cifras ubican a América Latina como importante mercado de la economía mundial (similar al de la República Federal de Alemania), aunque su capacidad de importación haya disminuido en los últimos años debido al drenaje de divisas que causa la deuda, y que agregado a la fuga de capitales y bajas tasas de inversiones, tiene un impacto deteriorante en la tasa de crecimiento económico. Para datos sobre PBI, ver: Progreso Económico Social. Informe Anual. Washington D. C., Banco Interamericano de Desarrollo (BID), varios años; y para datos sobre comercio exterior, ver Direction of Trade. Washington D. G., Fondo Monetario Internacional (FMI), varios años.
} 
tares; y b) América Latina en las percepciones o belief systems ${ }^{7}$ de los funcionarios gubernamentales y especialistas políticos de Estados Unidos.

Su principal contribución al primer punto reside en el hecho de analizar de una manera conjunta las múltiples generalizaciones que se hacen en torno a las posibles amenazas militares provenientes de América Latina, que en muchos casos han justificado políticas intervencionistas en aras de la estabilidad. Uno de los propósitos de Schoultz es analizar y contrastar con los hechos, de una manera sistemática y profundamente documentada, las aseveraciones en torno a la importancia estratégico-militar de América Latina para Estados Unidos. Recurriendo a documentos y declaraciones oficiales, a publicaciones de especialistas en la materia y a 260 entrevistas que a lo largo de ocho años realizó, Schoultz evalúa una por una las generalizaciones que aseveran que en la actualidad América Latina es estratégicamente importante para Estados Unidos. Las generalizaciones que Schoultz analiza son:

a) el acceso a materiales estratégicos en Latinoamérica es esencial para la seguridad de Estados Unidos;

b) el apoyo militar y las bases militares latinoamericanas son fundamentales para la seguridad de Estados Unidos;

c) el acceso a las vías marítima: de comunicación (sea lanes) es vital para Estados Unidos;

d) una base militar soviética en Latinoamérica conduciría a una modificación en el balance de poder que pondría en riesgo la paz mundial.

SchouItz hace un detallado análisis de cada una de estas generalizaciones y concluye que ninguna de éstas tiene bases reales. Según el autor, la importancia de América Latina en términos estratégicomilitares es, cuando mucho, modesta. Corresponde entonces preguntar epor qué continúan en Estados Unidos los grandes debates acerca de las amenazas a la seguridad nacional que supuestamente provendrían de Latinoamérica?

La respuesta a esa pregunta constituye una de las importantes contribuciones del trabajo de Schoultz. El autor nos introduce a un nivel de análisis hasta ahora muy poco explorado: el de las imágenes, los símbolos, las percepciones que filtran la realidad y que influyen sobre las decisiones de los funcionarios o especialistas poli-

TEI concepto de belief systems es tratado de manera amplia por Bruce Russet y Harvey Starr, World Politic. The Menu for choice (New York: W. H. Freeman and Company, 1985). Un análisis más detallado es realizado por Robert Jarvis, Perception and Misperceptions in international politics (Princeton, N. J.; University Press, 1976). 
ticos norteamericanos. Así resulta que la verdadera importanc:a estratégico-militar de América Latina se encuentra en las percepciones de los funcionarios y especialistas políticos. Éstos comparten una concepción "realista" del mundo, característica de la época de la Guerra Fría, según la cual, la paz mundial se sostiene en el balance de poder militar de las dos grandes potencias. Por lo tanto, la inestabilidad en una región considerada como su área de influencia significa para ellos una amenaza al balance de poder. El liderazgo de Estados Unidos se ve socavado y con ello se pone en peligro la paz mundial. De esta manera, el debate sobre Latinoamérica en Estados Unidos se convierte en un debate sobre las intencioncs de Ia Unión Soviética. Los países latinoamericanos son, desde esta perspectiva, sólo objetos en la lucha entre las dos superpotencias. Como lo revelan las entrevistas realizadas por Schoultz, ésta es una percepción muy extendida en el Gobierno de Reagan y entre politicos conservadores.

En su opinión, estas percepciones deberían modificarse, dado que a) el apoyo indiscriminado y el mantenimiento de dictaduras militares no es garantía de estabilidad y ocasiona mayores daños a lá seguridad de Estados Unidos, cuando estos regímenes invariablemente caen; b) Estados Un.dos no tiene ya la capacidad de imponer sus políticas unilateralmente, a un costo pequeño o moderado (e.g., Nicaragua y Panamá); y c) un tipo diferente de inestabilidad está empezando a surgir, la inestabilidad de las nuevas democracias.

El autor señala que ya existen indicios de que las concepciones "realistas", que correspondieron a otra época y a otro contexto internacional, están siendo reconsideradas debıdo principalmente a los nuevos problemas que están surgiendo en las relaciones entre Estados Unidos y las potencias medias latinoamericanas, que abarcan temas como la deuda, el proteccionismo comercial, la migración y el narcotráfico. Para la solución de estós problemas el "realismo" tradicional no tiene respuesta.

Schoultz se revela optimista al concluir su libro, afirmando que si bien los problemas de seguridad son todavia importantes en las percepciones de los funcionarios gubernamentales y especialistas políticos norteamericanos, su importancıa está declinando, porque reconocen que los problemas de Latinoamérica no pueden continuar siendo enmarcados en el contexto de la época de la Guerra Fría.

En términos generales, el libro de Lars Schoultz contribuye grandemente a refutar, con un análisis riguroso y detallado, aseveraciones que, al subrayar la importancia estratégico-militar de América Latina para la seguridad de Estados Unidos, obscurecen y desconocen el significado que esta región ha adquirido en otros ámbitos, lo que exige por tanto un tratamiento diferente, más complejo, más 
sofisticado, que el de simple objeto pasivo en el conflicto estratégico-militar entre las dos superpotencias ${ }^{8}$.

Bajo el sugestivo título de su libro Partners in Conflict. The United States and Latin America (Socios en Gonflicto. Estados Unidos y Latinoamérica), A. Lowenthal concentra su estudio en el análisis de las transformaciones económicas que ha experimentado América Latina a partir de la década de los sesenta. Su hipótesis fundamental es que como resultado del crecimiento económico de América Latina, han surgido nuevos temas en las relaciones interamericanas que plantean oportunidades para el confl:cto o la cooperación y por consiguiente, el autor considera que el futuro de estas relaciones dependerá del reconocimiento que Estados Unidos haga de esta transformación.

Sin negar las dificultades que todavía hay que superar en términos de distribución de la riqueza, Lowenthal elabora un detallado análisis de los cambios ocurridos en América Latina desde la década del 60 , en términos de población, de industrialización, de comercio exterior, de educación e inclusive de democratización politica. Al mismo tiempo que la región ha crecido, ha diversificado sus relaciones y se ha hecho más independiente, Estados Unidos ha tenido que enfrentar en ese período una creciente competencia económica y política que ha debilitado su pasición y su hegemonía mundial. Lowenthal considera que actualmente la presencia norteamericana en América Latina es similar a la que prevalecía en la época de Ios año; 20 y los 30 , caracterizada por ser extensiva pero sujeta a competencia por otras potencias. Agrega que si bien las asimetrías en las relaciones entre las dos regiones ha persistido, ésta se ha reducido durante los años 60 y los 70.

En este punto Lowenthal coincide con Schoultz en subrayar la importancia de las percepciones de los funcionarios gubernamentales en la formulación de la política exterior. Para el autor, la realidad latinoamericana ha cambiado en los últimos 25 años; pero los conceptos y las percepciones norteamericanas sobre el subcontinente

\footnotetext{
${ }^{3}$ Asimismo, el carácter de su investigación, basado en entrevistas $\mathrm{y}$ teniendo por objetivo el conocimiento de las percepciones de los funcionarios gubernamentales norteamericanos, nos permite entrar en un mundo generalmente vedado a los que habitan fuera de Washington. Sin embargo, al analizar las entrevistas, Schoulz no proporciona el porcentaje de los entrevistados que comparten esas opiniones o el área de la Administración en la que predominaban esas percepciones. Si bien es cierto que Schoulz lo señala con frases como "una gran parte" o "una minoría", el anńlisis se veria sin duda fortalecido si estuviera acompañado de cifras estadisticas que suministraran una idea más concreta de las afiliaciones ideológicas de los miembros de la presente administración. De csta manera, su estudio podria ser utilizado en futuras investigaciones en el que se analizaran de forma comparativa las percepciones que de América Latina tienen las sucesivas administraciones norteamericanas.
} 
no han evolucionado con igual rapidez. Es así que la Administración Reagan, según Lowenthal, no ha percibido lo mucho que Latinoamérica se ha transformado y por lo tanto no ha adecuado su política exterior a esa realidad diferente. Tras el análisis de los "intereses h'stóricos" por los cuales Latinoamérica ha sido tradicionalmente importante para Estados Unidos (bases militares, apoyo político, inversiones, materias primas), Lowenthal concluye que si la seguridad nacional norteamericana es definida en términos puramente militares, entonces, y como resultado de los avances tecnológicos, la importancia del subcontinente ha declinado ${ }^{9}$. Sin embargo, agrega, si el concepto de seguridad nacional se adecúa a una realidad transformada y se redefine como la "capacidad para proteger el bienestar individual y colectivo de los ciudadanos", entonces América Latina es vital para la seguridad nacional de Estados Unidos. Lowenthal coincide con Schoultz en que el concepto de seguridad nacional debe ser despojado de sus características puramente militares y debe incorporar elementos económicos, políticos $\mathrm{y}$ sociales que correspondan más a la actual realidad latinoamericana.

América Latina es ahora importante por los problemas que comparte con Estados Unidos (migración, deuda, narcotráfico, proteccionismo comercial, entre otros) y por la nueva naturaleza de las relaciones entre ambas subregiones. Problemas como migración, narcotráfico y deuda, que tienen fuertes repercusiones al interior de Estados Unidos, ya no pueden ser resueltos sin la cooperación de los países involucrados ${ }^{10}$.

Al contrario de lo que acontecía en la época de la postguerra, Estados Unidos ya no está en la posición, ni cuenta con la capacidad, para imponer unilateralmente una solución. Estos nuevos problemas exigen la cooperación de los países latinoamericanos. En palabras de Lowenthal: "cada voto (latinoamericano) debe ahora ganarse (lobbied for), ya no puede tomarse por seguro bajo la premisa de una 'upuesta 'armonía regional' "11. El autor agrega que la obsesión con el conflicto centroamericano ha obscurecido en las percepciones de los funcionario; gubernamentales de Ia Administra-

${ }^{\circ} \mathrm{La}$ superioridad tecnológica-militar de Estađos Unidos sobre América Latina y el balance estratégico-militar con la Unión Soviética han hecho obsbleta la noción de una posible amenaza militar a la seguridad de Estados Unidos pro. veniente de algún pais latinoamericano -al menos que ese país actúe como base militar de una potencia extracontinental, como hubiera sido posible durante la Primera o la Segunda Guerra Mundial.

${ }^{10}$ Estos nuevos temas en la agenda interamericana son analizados por un grupo de investigadores latinoamericanos y norteamericanos en Kevin J. Middlebrook y Carlos Rico (eds.), The United States and Latin America in the 1980's. op. cit.

IIVer capitulo III del libro de Lowenthal aquí analizado. 
ción Reagan la importancia de los problemas políticos y económicos del. resto de América Latina. Para Lowenthal, la amenaza a la segur dad nacional de Estados Unidos proviene de los nuevos problemas comunes y no de Centroamérica. Desde su perspectiva las potencias medias latinoamericanas y el Caribe son más importantes que ei conflicto centroamericano.

Por otra parte, la naturaleza de las relaciones económicas interamericanas ha cambiado, afirma Lowenthal. Mientras que en el pasado Estados Unidos podía aislarse del estado general de las economías latinoamericanas, pues sus relaciones económicas se limitaban a la extracción de recursos naturales, en la actualidad sus intereses económicos (inversión en la industria manufacturera, deuda, comercio) dependen del estado de la economía y de las finanzas en general de los países latinoamericanos. Para demostrar esta transformación ocurrida en las relaciones interamericanas, Lowenthal analiza separadamente los casos de México, Brasil y el Caribe, relevando Ia creciente "interpenetración" entre estos países y Estados Unidos en términos de migración, comercio, narcotráfico y deuda, entre otros.

Asimismo, Lowenthal hace la diferencia entre la "interpenetración" que caracteriza las relaciones entre Estados Unidos y Latinoamérica y el concepto más ampliamente difundido en los países industrializados conocido como "interdependencia"12. Al respecto, el autor señala que la "interpenetración" en los más diversos ámbitos entre Estados Unidos y (en este caso) México es evidente. Agrega que, sin embargo, ésta no puede ser considerada "interdependencia", en virtud de que el último concepto conlleva un sentido de equivalencia, similaridad o igualdad en la interconexión que en realidad no existe, al menos, subraya Lowenthal, no en las percepciones me$x^{i}$ canas, pues a pesar del crecimiento de México, el peso de Estados Unidos continúa siendo abrumador (overwhelming).

A diferencia de Schoultz, Lowenthal no sólo habla sobre la conveniencia de una nueva política norteamericana hacia Latinoamérica, sino que va más allá y propone una polftica general (no bilateral, ni exclusivamente Iatinoamericana) que enfrente las necesidades fundamentales de las potencias medias de América Latina y del Tercer Mundo. Según el autor, el apoyo norteamericano a reformas económicas internacionales (tales como el fortalecimiento de las instituciones financieras internacionales; promoción de nuevos préstamos comerciales a Latinoamérica con sobretasas menores; reducción del déficit fiscal $y$ de la tasa de interés norteamericana; expansión del comercio mundial), a la vez que permitiría a estas naciones reanudar el crecimiento y el desarrollo económico, repercutiría favo-

${ }^{12} \mathrm{El}$ concepto de interdependencia es ampliamente desarrollado en el libro dc Robert Keohane y Joseph Nye, Power and Interdependence: World Politics in Transition (Boston: Little Brown, 1977). 
rablemente en los mismos paises industrializados, debido precisamente al. fenómeno de la interpenetración.

Lowenthal es particularmente claro al subrayar la nueva importancia política y económica de los países medios de América Latina $y$ del Tercer Mundo en un nuevo sistema internacional que tiende a la multipolaridad. Si bien reconoce que Estados Unidos continúa siendo la nación más fuerte en el continente, el autor considera que su hegemonfa ha terminado, Ia distribución de poder ha camb:ado y el destino de los países industrializados no puede ahora desvincularse del de las potencias medias del Tercer Mundo. El autor acertadamente agrega que, a diferencia del pasado, las discrepancias con las potencias medias latinoamericanas pueden ahora convert $\mathrm{r}$. en un obstáculo formidable en la consecución de los objetivos internacionales de E-tados Unidos.

Finalmente, Lowenthal coincide con Schoultz al considerar que es tiempo de que Estados Unidos deje de ver a los países latinoam:ricanos como aliados automáticos y los reconozca como socios potenciales. Como re ultado de la declinación de su hegemonía y de las tran formaciones latinoamericanas, Estados Unidos no puede ya nrecumir, ordenar o ejercer coerción para obtener la cooperación de Latinoamérica. Por el contrario, en promoción de los propios intereses, Estados Unidos debe reconocer el fin de su hegemonía y promover la colaboración hemisférica para la resolución de problemas comunes. Lowenthal concluye que "si Washington puede abandonar los hábito de dominio. Étados Un'do- y Lat"noamérica pueden pasar del conflicto a la cooperación (partnership)".

Desde un punto de vista académico, Partners in Conflict es un libro menos riguroso que el libro de Schoultz. Si bien no es po-ible dudar de la aseveración de Lowenthal de que los indicadores económicos y sociales utilizados han sido revisados en varias ocasionex. la aurencia de notas no permite contrastar o refutar los datos.

Sin embargo, lo anterior no obscurece el amplio conocimiento que Lowenthal demuestra tener sobre América Latina. Particularmente, dos puntos sobresalen en su libro: la sensibilidad que manfiesta al tomar en cuenta en su análisis la perspectiva latinoamericana del problema, lo cual e:casea en los estudios que norteamericanos realizan sobre la región; y la propuesta de políticas alternat:vas, que prometen ser viables o al menos aceptables tanto para Estados Unidos como para América Latina en la búsqueda por una ntreva interrelación y una nueva inserción en un sistema internacional en continua tran'formación.

En realidad, más que pretender convertir su libro en uno de texto, Lowenthal parece querer convencer a los policy makers conservadores norteamericanos acerca de la necesidad de reconocer las 
transformaciones ocurridas tanto en Estados Unidos como en Latinoamérica; de cambiar actitudes y políticas heredadas de una época ya superada (la de incuestionable hegemonía norteamericana); y de buscar la cooperación con los países latinoamericanos en lugar de pretender su subordinación. Dado el alto costo psico-político y la magnitud de las concesiones que según los conservadores supondría el reconocimiento de la pérdida de hegemonía norteamericana, convencer a este sector norteamericano para que deseche sus costumbres o atavíos hegemónicos parece ser una tarea larga y difícil. En ese sentido, el libro de Lowenthal es un buen comienzo.

El libro editado por Grabendorff y Roett, Lat:n America, Western Europe, and the United States. Reevaluating the Atiantic Triangle, es el resultado de tres conferencias llevadas a cabo en 1981 y 1982 sobre el estado de las relaciones en el subsistema internacional denominado "triángulo atlántico", compuesto por Europa Occidental, Estado; Unidos y América Latina.

Hasta la fecha de edición (1984 en español, 1985 en inglés), muy pocas publicaciones habían abordado este tema. La abrumadora presencia de Estados Unidos en América Latina después de la Segunda Guerra Mundial opacó prácticamente el estudio de las relaciones entre el Viejo y el Nuevo Continente. Ia publicación de ecte libro viene a reflejar los cambios ocurridos en el sistema internacional, en el cual la potencia hegemónica occidental manifiesta signos de declinación, mientras que sus tradicionales zonas de influencia. incluyendo América Latina, se transforman, recuperando importantes márgenes de autonom ́a y exhibiendo un creciente activismo internacional.

Como lo mencionan los compiladores del libro, los cambios ocurridos en el "triángulo atlántico" exigen una reevaluación de las relaciones que lo conforman. Con este propósito, los colaboradores, principalmente especialistas europeos y latinoamericanos, exploran los alcances y límites de las crecientes relaciones europeo-Jatinoamericanas, así como las posibilidades de que su reforzamiento amplie los márgenes de negociación en ambas regiones y eventualmente conduzca a la instauración de in orden internacional más equitativo.

Los autores señalan en principio la intensidad que caracterizó las interacciones políticas y económicas entre América Latina y Europa en la época de la Preguerra y durante la Primera y Segunda Guerra Mundial. Subrayan que en el plano económico, América Latina era terreno en donde se dirimfan rivalidades de las grandes potencias europeas y americanas por ampliar y conservar mercados para sus productos manufacturados, préstamos e inversiones. América Latina era fuente importante de bienes de materias primas tales como trigo, carne, azúcar, café, tabaco, cobre, estaño, caucho, 
entre otras, que resultaban vitales para las potencias europeas durante la Primera y Segunda Guerra Mundial ${ }^{13}$.

En lo estratégico militar, la región era igual de importante. América Latina y el Caribe fueron territorios de interés estratégico-militar para las potencias europeas y en varias ocasiones fueron arena de conflicto entre ellas y Estados Unidos. En ambas regiones, durante el siglo pasado, Gran Bretaña disputaba a Estados Unidos el predominio y el control militar y económico de las mismas; y durante las dos guerras mundiales, Alemania intentó penetrar la región para desafiar el creciente poder hegemónico de Estados Unidos.

El crecimiento económico y los esfuerzos expansionistas estadounidenses; la declinación económica y militar de Europa, principalmente de la potencia hegemónica, el Reino Unido; la derrota del régimen alemán al final de la Segunda Guerra; y la instauración de un sistema internacional basado en el liderazgo indiscut:ble de Estados Unidos en el hemisferio occidental, fueron los factores esenciales que determinaron el deterioro de las relaciones políticas, económicas y estratégico-militares entre Europa y América Latina.

Desde el fin de la Segunda Guerra Mundial hasta principios de la década del setenta, los pocos intentos de acercamiento entre ambas regiones (tales como el "De Gaullismo") fracasaron. No es hasta la crisis de los energéticos de la década de los 70 que Europa mostró de nuevo su interés en el subcontinente. Las reservas de hidrocarburos en la región; el crecimiento de los mercados de las potencias medias como Brasil, México o Argentina; la distensión internacional, son Ios principales factores señalados por los autores como los motores del nuevo intento europeo por acercarse a Latinoamérica. A partir de entonces Francia, la República Federal Alemana, la Comunidad Económica Europea, la Internacional Socialista y la Democracia Cristiana Internacional principalmente, iniciaron una nueva ofensiva en la región.

Desde la perspectiva latinoamericana, los autores analizan exhaustivamente el caso de Brasil como pionero en el acercamiento europeo-latinoamericano. Este país, en su intento por reducir su dependencia económica, inició en la década del 60 una polf́tica exterior basada en la diversificación de sus relaciones económicas y comerciales, principalmente hacia Europa Occidental. Sin embargo, señalan Ios autores, Brasil no intentó substituir a Estados Unidos por Europa, sino utilizar la rivalidad económica entre ambas regiones para aumentar su poder de negociación en el sistema interna-

\footnotetext{
${ }^{13}$ Sobre el importante papel económico que Latinoamérica jugó en el sistema internacional en la época de las dos guerras mundiales, consultar el excelente trabajo de Rosemary Thorp, Latin America in the 1930's. The Role of the Periphery in World Crisis (New York: St. Martin's Press, 1984).
} 
cional. Los casos del desarrollo de la energía nuclear y la industria de armamentos en Brasil son particularmente ilustrativos de lo anterior. Esta política, que demuestra una mayor "agresividad" (assertizueness) en su formulación y ejecución no fue un fenómeno aislado, considera Carlos Moneta, uno de los autores de este libro, y su ejemplo fue seguido, aunque en menor medida, por algunas potencias medias como Argentina, Venezuela y México.

Aquí conviene subrayar la coincidencia existente entre los autores analizados: son las potencias medias de América Latina y no todo el subcontinente las que están ocupanḍo un nuevo lugar en el sistema internacional. Son ellas las que con sus mercados más amplios, sus deudas de mayor magnitud y sus mayores recursos son capaces de influir en el sistema internacional, y son ellas las que Europa empezó a "cortejar" en su acercamiento a Latinoamérica. Grabendorff, en su introducción, explícitamente reconoce que el libro re concentra en el estudio de los países latinoamericanos más grandes. El artículo de Roberto Russell contribuye particularmente a ahondar en este tema, señalando la diferenciación que actualmente está ocurriendo entre los países subdesarrollados y que está dando lugar al surgimiento de una "clase media internacional" compue-ta oor potencias medias tales como India, México, Brasil, Argentina y Venezuela, con recursos e intereres cada vez más diferentes al de los países menores.

Igualmente, los autores coinciden en señalar que los gobiernos de las potencias medias de Latinoamérica se han dado cuenta de su capacidad disruptiva y constructiva en el sistema internacional, lo que consecuentemente ha incrementado su poder de negociación $y$ su importancia en este sistema. Moneta, afirma particularmente que el nuevo "status" de los países medios latinoamericanos resultó p.o sólo de su crecimiento económico, sino también del desarrollo de una política más "agresiva" (assertive) en el terreno internacional y de su voluntad de desafiar el sistema internacional establecido, en búsqueda de su propio interés nacional o regional. Muestra de esta nueva actitud ha sido el liderazgo ejercido deside los setenta por las potencias medias latinoamericanas en la promoción de un Nuevo Orden Internacional en el seno de la ONU, en la UNCTAD, en el Grupo de los 77 y la creación del sela, de la ALADI y de Contadora.

Grabendorff coincide con Moneta y agrega que los gobiernos latinoamericanos han demostrado gran habilidad para incrementar su poder de negociación frente a Estados Unidos a través de la "globalización" de problemas regionales y de la diversificación de sus relaciones, no sólo hacia Europa sino hacia otras regiones.

Sin embargo, los colaboradores del libro de Grabendorff-Roett 
consideran que lo anterior no podría ser explicado sin la declinación hegemónica de Estados Unidos. En ese sentido, Roett, quien concluye el libro, coincide con Schoultz y Lowenthal en subrayar que Estados Unidos ya no es capaz de dar unilateralmente una solución a los problemas de Latinoamérica y agrega que ahora se ha convertido en parte del problema, como en el caso de Centroamérica. Al igual que los autores de los dos libros previamente analizados, Roett atribuye a las dificultades en las relaciones interamericanas a la inhabilidad de Estados Unidos para percibir y reconocer los cambios ocurridos en Latinoamérica. Según Roett, esto se debe a que las percepciones sobre el subcontinente e-tán todavía obscurecidas por las consideraciones estratégico-militares, debido al "tremendo shock que la crisis de misiles en Cuba dejó en las mentes de los policy makers norteamericanos" 14 .

Haciendo referencia a la creciente "interdependencia" que caracteriza las actuales relaciones internacionales, Roett agrega que, "a diferencia de la época de la indisputable hegemonía norteamericana, el futuro de Estados Unido; está ahora ligado a la cooperación que obtenga tanto de Europa como de Latinoamérica y que si bien el estrechamiento de lazos entre estas dos regiones puede causar confusión en Estados Unidos, finalmente éste viene siendo útil en virtud de que incrementa las opciones politicas y económicas de Latinoamérica".

Por otra parte, si bien los autores se manifiestan optimistas sobre Ias potencialidades del acercamiento entre Europa y América Iatina para el reforzamiento de la autonomía de los subsistemas regionales y particularmente el poder de negociación de Latinoamérica en el sistema internacional, coinciden en señalar que hasta ahora el reencuentro de estas dos regiones ha sido tímido y con muchos obstáculos. Las principales dificultades que los autores señalan, emanan de las diferentes expectativas que ambas regiones tienen sobre la relación europeo-latinoamericana, lo que marca sus alcances y sus lfmites. Mientras que para Europa, ésta tiene significados eminentemente económicos dada su "dependencia estratégica" en Estados Unidos, para América Latina, la nueva relación tiene una fuerte connotación politica en virtud de sus posibilidades para reducir su dependencia e incrementar su "autonomía periférica"15. Esto es, mientras que los esfuerzos europeos no tienen por fin la modificación del orden mundial, los intentos latinoamericanos por otro lado cuestionan abiertamente el "statu quo" internacional.

${ }^{14}$ Ver, R. Roett, p. 267, del libro aquf analizado.

${ }^{25}$ Sobre el concepto de autonomia periférica, ver Helio Jaguaribe, "Hegemonía Céntrica y Autonomía Periférica", en: E. Hill y L. Tomassini (comp.), América Latina y el Nuevo Orden Económico Internacional, op. cit. 
Por último, los autores concluyen que la nueva importancia de Latinoamérica (más precisamente, de las potencias medias) en el "triángulo atlántico" y en el sistema internacional, va a causar inevitablemente confusión e inestabilidad. Sin embargo, agregan que la consolidación del actual sistema internacional que tiende a la multipolar:dad y a la interdependencia pasa por la aceptación de Latinoamérica como parte activa e importante de este sistema. De esta región, concluyen, ya no se puede esperar un "alineamiento automático". Latinoamcrica va a defender sus propios intereses y valores que al fin y al cabo son los mismos de los otros dos miembros de! "triángulo atlánt co". L os autores concluyen que "Latinoamérica seguirá siendo parte de Occidente, pero ya no como prisionera de Occidente".

El retraimiento de Luropa Occidental a partir del ascenso de la Administración Reaģan, ciemostró que los autores del libru editado por Grabendorff y Roet estaban correctos en su cautela respecto a las limitaciones de ias relaciones europeo-latinoamericanas. La nue va política exterior norteamericana, que tiene como fundamento precisamente revertir o al menos detencr el procero de declinación hegemónica de Estados Unidos, significó en la práctica la redefinición de su tradicional zona de influencia y la reducción de influencias extrañas en la región, incluyendo las de carácter moderado como e! movimiento social demócrata internacional.

Sin embargo, si bien el retraimiento europeo implicaba lógicamente una reducción de la capacidad de Latinoamérica para influir en la escena internacional, los hechos posteriores a 1983 demostraron que la presencia europea, aunque deseable, no es indispensable en el fortalecimiento de la posición negociadora del subcontinente. El acelerado incremento en las consultas regionales, la creación del Grupo Contadora, el Consenso de Cartagena, la reunión de Presidentes del Grupo de los Ocho, los proyectos de integración Argentina-Brasil, Uruguay-Brasil, entre otros, así lo confirman.

En este sentido, el aumento de la importancia de América Latina en la escena mundial -a pesar de las dificultades económicas o precisamente debido a ellas- difícilmente puede ser visto como un evento puramente coyuntural o como resultado exclusivamente del incremento de los lazos con Europa Occidental o de la declinación hegemónica de Estados Unidos. El aumento del poder negociador de Latinoamérica revela así que su importancia en el sistema internacional está más directamente relacionada a su transformación, a su crecimiento económico y a su voluntad política para defender sus intereses nacionales y regionales en la comunidad internacional.

El libro editado por Carlos Ominami, La Tercera Revolución Industrial, Impactos Internacionales del Actual Viraje Tecnológico, 
trata de las posibilidades, las opciones y los desafíos que le ofrece el futuro a América Latina como consecuencia de los cambios tecnológicos en el sistema internacional.

Sin caer en la especulación, especialisitas en la materia abordan temas tan aparentemente distantes y extraños a la realidad latinoamericana como la microelectrónica, la biotecnología, los superconductores. La tercera revolución industrial está en marcha y está provocando una reordenación en la jerarquía internacional. Los principales puntos de discusión en el libro se refieren a los impactos de la transformación tecnológica en el proceso de desarrollo latinoamericano y al lugar que ocupará América Latina en el nuevo sistema internacional en formación.

La tercera revolución industrial está caracterizada por la instauración de un nuevo paradigma tecnológico que transforma significativamente la organización productiva y el sistema económ:co internacional. Como afirma una de las colaboradoras, Carlota Pérez, este nuevo paradigma (la microelectrónica), está basado ya no en la existencia de recursos energéticos baratos, sino en la tendencia a la automatización de los procesos productivos y a la disponibilidad de información barata y abundante. En la práctica, lo anterior significa que debido a la instauración del nuevo paradigma tecnológico, la abundancia o no de recursos naturales (rubros en los cuales América Latina gozaba internacionalmente de ciertas ventajas comparativas) deja de ser un factor económico importante.

Los trabajos de Paulo Bastos, Gonzalo Arroyo y Juanita Gana revelan los efectos que el viraje tecnológico internacional tiene sobre el sistema económico latinoamericano. En la rama de la electrónica, Paulo Bastos analiza cómo los nuevos sistemas de computación, de automatización industrial y de robotización plantean a Latinoamérica serios desafíos económicos y sociales, de los cuales los más importantes son los altos niveles de educación requeridos y los efectos negativos que podrían tener en el empleo.

En la cuestión de la biotecnología, los nuevos adelantos científicos, según Gonzalo Arroyo, hacen prever la drástica disminución de utilización de materias primas agrícolas de las cuales América Latina tiene abundancia. El caso del azúcar, cuya demanda ha caído significativamente como resultado de la utilización de las isoglucosas, es sumamente ilustrativo de lo que puede ser el futuro de la mayoría de las materias primas latinoamericanas.

El descubrimiento de nuevos materiales, tales como los superconductores, fue resultado del temor que prevaleció en los setentas de un eventual agotamiento de materias primas, afirma Juanita Gana. Lo anterior ha ocasionado tamb én la fuerte caída del consumo y el precio de metales tradicionalmente latinoamericanos tales como 
el cobre y el estaño, con efectos particularmente dañinos para parses tales como Perú, Bolivia y Chile. Dado el acelerado ritmo de sustitución de materias primas por materiales sintéticos al que conduce la tercera revolución industrial, las proyecciones muestran una tendencia a la declinación significativa del uso de los metales tradicionales en los años futuro: Si bien esto resulta positivo desde el punto de vista de la conservación de los recursos naturales, plantea serias dificultades a los diversos países latinoamericanos que han basado su economía en la producción y exportación de metales.

¿Significa, entonces, que en términos generales la revolución tecnológica y sus implicaciones son una amenaza para el desarrollo económico latinoamericano? La opinión de los autores coincide en que a la vez que ofrece peligros y riesgos para las tradicionales bases económicas latinoamericanas (abundancia de mano de obra s, recursos naturales), también ofrece la oportunidad de colocar al subcontinente en una mejor posición en la nueva división internacional del trabajo. Esto sería así únicamente si se adoptan políticas adecuadas de absorción y adaptación tecnológica, principalmente en el área de formación de recursos humanos y de investigación y desarrollo ( $\&$ \& $D$ ).

Los nuevos descubrimientos tecnológicos pueden así transformarse de amenaza, en palanca catalizadora para el desarrollo latinoamericano. La flexibilidad en la organización industrial que caracteriza la utilización de la microelectrónica, ofrece la ventaja de eliminar la necesidad de economías de escala, que ha sido hasta hoy una de las principales limitantes de las naciones subdesarrolladas para producir a precios competitivos. Por otra parte, la microelectrónica y el uso del software, permite una absorción más rápida de tecnologías nuevas por parte de los países subdesarrollados, al contrario de lo que sucedía en el pasado, cuando la tecnología que llegaba a estos países estaba en su fase madura o era francamente obsoleta.

En el terreno de la biotecnología, ésta puede ser utilizada, según Ios autores, para enfrentar el hambre y la desnutrición en las naciones subdesarrolladas a través de la solución de problemas tales como la aridez, salinidad y alcalinidad de la tierra, entre otros.

En suma, si los gobernantes latinoamericanos deciden fomentar abiertamente la tecnología, el nuevo viraje tecnológico puede constituirse no sólo en un obstáculo sino en un instrumento de desarrollo nacional. La diferencia del papel de la tecnología en América Latina, como amenaza o palanca de desarrollo, dependerá definitivamente de los esfuerzos que se hagan para preparar los recursos humanos y concentrar los económicos en la adopción de la nueva tecnología, . . . 
Sin embargo, las actuales cifras indican que a Latinoamérica le falta todavía un largo camino por recorrer. La región (con la excepción de Brasil) se ha caracterizado precisamente por su atraso en educación e investigación tecnológica. Las cifras que al respecto menciona en su artículo Francisco Sagasti son francamente alarmantes. Según la unesco, en 1980 América Latina contribuyó sólo con el $1,8 \%$ del gasto total mundial en investigación científica, mientras que dedicó sólo el $0,69 \%$ del PNB regional en este tipo de actividades. La cifra resulta ilustrativa si re compara con los gastos anuales de Europa, Estados Unidos y Japón, que oscilan entre el 2,6 al 2,9\% de su PNB.

Finalmente, el análisis del caso de Brasil realizado por Fabio $\mathbf{S}$. Eerber, muestra tanto las posibilidades como lo: límites del avance tecnológico. En términos estrictamente económicos resulta impresionante el avance logrado por este país en las ramas de la aeronáutica, la informática, la energía nuclear y las telecomunicaciones. Sin embargo, la adopción de estas políticas sin un objetivo claro de desarrollo interno impidió que el salto tecnológico ayudara a reducir los grave problemas de desempleo y de servicios públicos tales como salud, higiene, educación y transporte urbano que caracterizan a Brasil y en' general a las naciones latinoamericanas.

Una lección que parece desprenderse del caso de Brasil es que el avance tecnológico no equivale a desarrollo. Para hacer coincidir tecnología y desarrollo se requiere la participación activa de la población en el nuevo proyecto económico, sin la cual la tecnologia pierde su capacidad liberadora y perpetúa los esquemas inequitativos que tradicionalmente han caracterizado a América Latina.

El libro compilado por Ominami contribuye sin duda a despejar el oscuro tema del impacto de la revolución tecnológica en América Latina. Sin embargo, vale la pena destacar dos puntos no desarrollados ampliamente en el libro.

Los autores coinciden en que los diferentes niveles de desarrollo relativo entre los países de América Latina son cruciales. La capacidad para incorporarse con una mejor posición en la jerarquía internacional que resulte de la tercera revolución industrial, dependerá en mucho de la dotación de recursoi humanos capacitados para absorber y adaptar las nuevas tecnologías. Esto es, las potencias medias de Latinoamérica tienen una ventaja sobre los países pequeños en cuanto a su mayor capacidad de adaptación tecnológica. Lo anterior sugiere importantes preguntas que no fueron planteadas en profundidad por los autores: tla introducc ón de nueva tecnología va a incrementar el proceso de diferenciación entre las potencias medias y los paises menores? ¿Esta diferenciación conspirará contra la adopción de una postura conjunta de los países latinoamericano 
en las negociaciones económicas internacionales? ¿Es posible hablat de una América Latina? ¿O en realidad lo que se observa es la tendencia a la formación de dos América Latina: las potencias medias y los países pequeños? $\mathrm{Si}$ estos últimos tienen reducidas posibilida des de absorber los avances tecnológicos por la escasez de recursos, ¿es posible incorporarlos en una estrategia regional de desarrollo tecnológico, o se los estará condenando a mantenerse al margen de los potenciales beneficios de la nueva transformación tecnológica?

El segundo punto se refiere a la necesidad de reevaluar los organismos internacionales y la cooperación internacional como instrumentos indispensables en desarrollo tecnológico. Los enormes recursos que se necesitan para la investigación y el desarrollo tecnológico difícilmente pueden ser absorbidos individualmente por los paises subdesarrollados. Que la mejor forma de enfrentar el desafío tecnológico es la cooperación internacional, lo demuestra el hecho de que inclusive los países desarrollados que cuentan con mayores recursos han optado por esta vía. Tal es el caso del proyecto coniunto europeo "Eureka", los convenios tecnológicos entre Europa Occidental y Japón, Estados Unidos y Japón. y el Sistema de Defensa Estratégico norteamericano.

Organismos regionales de cooperación como la OEA y el SELA deberían ser fortalecidos para jugar un papel más importante como promotores del desarrollo científico tecnológico de los países de? área, y en particular de los menos desarrollados; y como catalizadores y facilitadores de una mayor cooperación entre los países más desarrollados y los menos desarrollados.

\section{ConClusrón:}

Los libros aquí analizados concuerdan en destacar que, en comparación a los sistemas internacionales de la preguerra y la postguerra, la importancia de América Latina en términos estratégico-militares ha disminuido, mientras que en el campo económico político su importancia ha aumentado. Es decir, como resultado de los cambios ocurridos en el contexto mundial y regional, América Latina se está convirtiendo en un actor capaz de ejercer cierta influencia política y económica en ambos niveles.

Si bien en la preguerra y postguerra, América Latina era un actor pasivo en el sistema internacional (con la posible excepción de Argentina), en la actualidad las potencias medias del subcontinente constituyen un activo grupa de participantes en el sistema internacional, con creciente autonomía y capacidad de influencia en los asuntos internacionales. Esto se ha debido fundamentalmente a tres 
factores: 1) El cambio tecnológico, que redujo drásticamente el significado estratégico-militar de la proximidad geográfica de América Latina a una de las superpotencias; 2) la declinación de la hegemonía de Eștados Unidos, que abrió espacios políticos y económicos en favor de otras naciones; y 3) la transformación y desarrollo de países y regiones anteriormente dependientes de Estados. Unidos (Japón, Europa Occidental y América Latina) al grado de cuestionar la hegemonfa norteamericana.

Las cuatro publicaciones aquí analizadas han coincidido en señalar los significativos avances en los campos políticos y económicos que ha experimentado América Latina (principalmente las potencias medias) en el sistema internacional, en las últimas décadas. Sin embargo, los autores coinciden también en que este no es un hecho definitivo ni irreversible. Factores tales como los intentos de reafirmación hegemónica de la actual administración en Estados Unidos, la crisis de la deuda y el desafío tecnológico amenazan con detener ese progreso o hacer retroceder a América Latina de las posiciones ganadas.

En ese contexto de desafíos y posibilidades, la solidaridad, la cooperación regional y las estrategias de negociación conjuntas se prevén como elementos fundamentales para la defensa y promoción de los intereses y valores de la región en el sistema internacional. La época en que los gobiernos latinoamericanos tendían a diferenciar entre política nacional e internacional, con negligencia hacia esta última, en que un füerte nacionalismo reducía la capacidad de negociación Iatinoamericana, hoy día parece estar superándose en aras de una mayor coordinación de las políticas exteriores.

Tal como lo expresan los libros aquí analizados, las transformaciones ocurridas en América Latina y, como consecuencia, la influencia que ella va adquiriendo en los asuntos internacionales se comienza a reconocer explícitamente. Paralelamente, ya se aprecian signos de que los gobiernos de la región están actuando cada vez más en consonancia con esos cambios y esa nueva realidad. El Grupo de Contadora, el Grupo de los Ocho, el Consenso de Cartagena y los pactos de integración entre Argentina, Uxuguay y Brasil son claros ejemplos de esa tendencia. Pero el futuro de la región latinoamericana en el sistema internacional dependerá mucho de que estos nuevos mecanismos de coordinación regional, se profundicen $y$ se reproduzcan en todo el subcontinente. Por el momento, el desafío de las crisis internacionales que afectan vitalmente a América Latina está conduciendo a los nuevos líderes latinoamericanos a poner en práctica lo que desde hace varias décadas se encontraba en la retorica del papel: la cooperáción y la integración regional.

Por otro lado, es alentador observar la creciente importancia y

$$
\text { [ } 20 \% 4]
$$


relevancia que va adquiriendo en círculos académicos, gubernamentales, diplomáticos y económicos de América Latina el estudio sistemático y riguroso de las relaciones internacionales de la región. El estudio y las investigaciones sobre las relaciones internacionales de la misma es fundamental para la generación endógena de conocimiento e información sobre las estructuras, los cambios y las tendencias internacionales que condicionan el proceso de desarrollo y de integración de nuestros países; como lo es también la formación de profesionales con un entendimiento adecuado del impacto que los fenómenos internacionales tienen sobre nuestras sociedades. Cada día es más evidente la necesidad que nuestros pafses tienen de contar con expertos en asuntos internacionales que puedan contribuir a formular políticas públicas realistas de acercamiento y cooperación, que respondan efectivamente a los desafíos de la interdependencia $y$ las transformaciones internacionales ${ }^{16}$.

No aprovechar las nuevas oportunidades para la cooperación y la integración regional en la actualidad no sólo debilitaría la posición internacional de las naciones latinoamericanas sino que tambión, como afirma Ominami, obligaría a Latinoamérica a realizar "una penosa retirada de la Historia".

BIBIIOGRAFIA

LARS ScHoulT, National Security and United States Policy toward Latin America. Princeton, N. Y.: Princeton University Press, 1987.

Abrafam Lowenthal, Partners in Conflict. The United States and Latin America. Baltimore, Md.: The Johns Hopkins University Press, 1987.

WOLF GRABENDORFF Y RIORDAN ROETT (eds.), Latin America, Western Europe and the United States. Revaluating the Atlantic Triangle. New York, N. Y.: Praeger, 1985.

Garlos OmINAmI (ed.), La Tercera Revolucion Industrial. Impactos Intemacionales del Actual Viraje Tecnológico. Bs. As., Argentina: Grupo Editor Latinoamericano, 1986.

10Ver Rubén M. Perina (comp.), El Estudio de las Relaciones Internacionales en América Latina y. él Caribe (Buenos "Aires: GEL, 1985); L. Tomassini, "El Análisis de: la Política Exterior en un Mundo más Complejo". Santiago, mimeo, Rust, 1986; y Carlos J. Moneta; "Modernización. y Politica Exterior en América Latina". Caracas, mimeo rNvesP, 1986. 